Socio-anthropologie et auto-promotion communautaire dans la région d'Itombwe : pour une stratégie de l'espoir

\title{
Kaza Rutereza
}

\section{CpenEdition}

\section{Journals}

Édition électronique

URL : http://journals.openedition.org/apad/391

DOI : 10.4000/apad.391

ISSN : 1950-6929

Éditeur

LIT Verlag

\section{Édition imprimée}

Date de publication : 15 mars 1992

\section{Référence électronique}

Kaza Rutereza, « Socio-anthropologie et auto-promotion communautaire dans la région d'Itombwe pour une stratégie de l'espoir », Bulletin de l'APAD [En ligne], 3 | 1992, mis en ligne le 06 juillet 2006, consulté le 08 septembre 2020. URL : http://journals.openedition.org/apad/391 ; DOI : https://doi.org/ 10.4000/apad.391

Ce document a été généré automatiquement le 8 septembre 2020.

Bulletin de I'APAD 


\title{
Socio-anthropologie et auto-promotion communautaire dans la région d'Itombwe : pour une stratégie de l'espoir
}

\author{
Kaza Rutereza
}

1 La région d'Itombwe-Sud (Zaïre) est l'une des plus défavorisées au Monde de par son enclavement. Mais par contre, il existe un potentiel économique riche et énorme encore inexploité. La population en prend de plus en plus conscience et tente d'y réagir mais en vain faute d'un appui fort et avisé. En tant que cible de nos investigations, cette région offre un cadre référentiel par excellence pour les études des communautés rurales en pleine mutation. Elle est généralement hissée au rang de grenier de la région des grands lacs (Burundi, Rwanda, Zaïre-Est).

2 Sa spécificité est une co-existence pacifique de deux types d'économie, l'une agricole et l'autre pastorale. L'on pourrait d'ailleurs parler de deux procès de production économique dans une diversité culturelle.(...). En dépit des déchirements socio-politiques et culturels, résultats de la dérive politico-économique nationale, les populations d'Itombwe se sont résolues à travers le processus de socialisation à la base à créer des initiatives locales de développement en vue de s'attaquer à la croissante paupérisation et dégradation de l'environnement qui est à l'origine de la crise agraire dans la région. Une étude-diagnostic vient d'être menée par un membre de l'APAD afin de mieux cerner la situation : 20 initiatives locales ont été dénombrées. Elles ont pour domaines d'activités: l'agriculture, l'élevage, l'environnement, l'hydraulique et l'assainissement, l'alphabétisation fonctionnelle, la santé et l'habitat, l'amélioration des pistes, l'éducation, etc. Ces initiatives dont l'âge moyen est de deux ans connaissent pour la plupart un bilan quasi-négatif de par leur degré d'auto-propulsion et d'auto-développement. Des marches-arrières sont mêmes visibles pour la majorité d'entre elles, faute d'un appui fort et avisé consistant à renforcer leurs capacités d'auto-promotion. La dégénérescence de ces initiatives est due à une pluralité de causes 
dont les origines sont à la fois structurelles (clanisme, incompétence, méconnaissance générale) et conjoncturelles (faiblesse des fonds de roulement, absence d'appui financier extérieur).

3 En dépit de ces blocages, de nouvelles stratégies d'espoir viennent d'être identifiées afin de faciliter le décollage effectif du développement à la base. Il s'agit des stratégies basées sur la valorisation des ressources locales et la promotion des activités réalisables et durables (économiques, techniques, sociaux et juridiques), ainsi que la recherche-accompagnement et l'appui-institutionnel en vue de soutenir l'expérience en marche et déboucher sur la création d'un réseau d'entreprises locales durables et crédibles.

4 Cette offre des savoirs anthropologiques augure une nouvelle ère dans la problématique du développement rural dans la région. C'est toutefois à titre marginal que cette intervention trouve place dans un dialogue de sourds "développeurs-développés". Ce faisant, l'anthropologue, dans sa dimension d'appuyeur, mieux, d'accompagnateur, gagnerait à adopter une attitude d'éclaireur dans toutes les phases d'un projet ou programme de développement en se constituant en un trait-d'union entre développeurs et bénéficiaires-acteurs. Ce qui permet d'atténuer ou de supprimer la dépendance des seconds envers les premiers en créant une nouvelle éthique basée sur un partenariat juste et équitable. (...) 